\title{
Enseñanza basada en proyectos: una forma alternativa para enseñar Álgebra Lineal ${ }^{*}$
}

\author{
Judit Taberna $^{1}$ y Maria Isabel García-Planas ${ }^{1}$ \\ ${ }^{1}$ Universitat Politècnica de Catalunya
}

\begin{abstract}
In order to promote the commitment and motivation of engineering students in linear algebra we have proposed a Project-based learning, giving a dynamic classroom approach in which students modeled real-world problems and turn gain a deeper knowledge of the subject. Considering that most students are digital natives, we use the e-portfolio as a tool of communication between students and student-teacher. In this article, we present an overview of the design and implementation of a project-based learning for a linear algebra course taught at the "ETSEIB" of Universitat Politècnica de Catalunya (UPC).
\end{abstract}

Keywords: project-based learning, e-portfolio, linear algebra.

\section{Resumen}

Con el fin de promover el compromiso y la motivación de los estudiantes de ingeniería en la asignatura de álgebra lineal, hemos propuesto un aprendizaje basado en proyectos, dando un enfoque de clase dinámica en la que los estudiantes modelan problemas del mundo real con el fin de obtener un conocimiento más profundo de la materia. Teniendo en cuenta que la mayoría de los estudiantes son nativos digitales, utilizamos el e-portafolio como herramienta de comunicación entre los estudiantes y el maestroalumno. En este artículo, presentamos una visión general del diseño y la implementación de un aprendizaje basado en proyectos para un curso de álgebra lineal enseñado en la "ETSEIB" de la Universitat Politècnica de Catalunya $(U P C)$.

Keywords: aprendizaje basado en proyectos, e-portafolio, álgebra lineal. 


\section{Introducción}

Es bien sabido que el Álgebra Lineal es un tema básico en diferentes áreas de las ciencias. Esto es debido a los múltiples problemas que se pueden modelar utilizando sistemas lineales cuyas soluciones se pueden obtener fundamentalmente a través del Álgebra Lineal. Sin embargo, una de las principales dificultades de los estudiantes de primer año de universidad matriculados en grados de carreras científicas o técnicas diferentes del grado de Matemáticas es que no ven la importancia que las matemáticas, en general, y del Álgebra Lineal en particular, pueden tener en sus campos de estudio y futuro profesional. Esto puede afectar seriamente a la motivación de los estudiantes en el curso, e impedir su éxito. Este efecto parece ser más pronunciado en el primer año de álgebra lineal, debido al factor abstracto de la materia, al contrario que por ejemplo la materia de cálculo que les es más fácil pensar que les puede ser útil.

Desde los años 90, se está tratando de reformar la manera de enseñar Álgebra lineal. Concretamente, en 1993, en los EE.UU, el grupo "Linear Algebra Curriculum Study Group (ICSG)"publicó una serie de recomendaciones para la impartición de un curso de Álgebra Lineal debido a que se dieron cuenta de que en muchas escuelas el plan de estudios de Álgebra Lineal" no aborda adecuadamente las necesidades de los estudiantes" (Carlson and Al. (1993)). Con estas recomendaciones, no sólo se estimuló la investigación de la enseñanza de álgebra lineal, sino también se publicaron una amplia lista de artículos sobre este tema (véase Yildiz (2013) y Aydin (2009), por ejemplo). Entre estos estudios, cabe destacar lo que varios investigadores dicen sobre el uso de las TIC, y cómo afecta a las actitudes tanto de los estudiantes y a sus logros académicos.

Siguiendo la teoría psicológica de Piaget sobre el desarrollo del concepto, Harel en (Harel (2000)) destacó los siguientes tres "principios" para la enseñanza del álgebra lineal: la concreción, la necesidad y la posibilidad de generalizar. El principio de concreción afirma que: "Para que los estudiantes puedan extraer una estructura matemática de un modelo dado de dicha estructura, los elementos de este modelo deben ser entidades conceptuales a los ojos del estudiante". Es decir el estudiante tiene los procedimientos mentales que pueden tomar como entradas de estos objetos. En cuanto al Principio de necesidad "los estudiantes deben ver una necesidad intectual de lo que están destinados a ser enseñados". Por último, el Principio de generalizar dice que "cuando la instrucción se refiere a un modelo 'concreto', es decir, un modelo que satisface el principio concreción, las actividades de instrucción dentro de este modelo deben permitir y fomentar la generalización de los conceptos".

En la búsqueda de alternativas para mejorar la enseñanza y el aprendizaje de Álgebra Lineal, diferentes experimentos se han diseñado e implementado. Se ha llegado a la conclusión que para mejorar la enseñanza y el aprendizaje del Álgebra Lineal puede ser útil incluir alternativas, como el uso de nuevas tecnologías, en concreto, por medio de la creación de e-portafolios y la ejecución de proyectos tanto de forma individual y en grupos. 
Por esa razón, el objetivo de esta investigación es analizar los beneficios del aprendizaje basado en proyectos (PBL) en el plan de estudios de Álgebra Lineal mediante la creación de e-portafolios en grupos grandes, como herramienta para la enseñanza, el aprendizaje, la evaluación y la autoevaluación de aprendizaje de los estudiantes. El PBL consiste en que los alumnos de forma colaborativa y activa planeen, desarrollen y evalúen un proyecto de trabajo de aplicación práctica. Esto conlleva la propuesta al grupo de estudiantes de la resolución de problemas o de cuestiones complejas para las que deben diseñar un plan de trabajo, ponerlo en práctica e ir tomando decisiones durante la aplicación a la vez que ir resolviendo los problemas que van surgiendo (Márquez, E. y Jiménez-Rodrigo, M.L. (2014)).

Al final del artículo, mostramos los resultados de este estudio, la satisfacción, así como la utilidad de esta herramienta. Los participantes utilizados para este proyecto son todos los estudiantes del primer año de Ingeniería en la asignatura de Álgebra lineal en la Escola Tècnica Superior d'Enginyeria Industrial de Barcelona (ETSEIB) de la Universitat Politècnica de Catalunya (UPC).

\section{Características del concepto de aprendizaje basado en proyectos}

El aprendizaje basado en proyectos (PBL) es un modelo que organiza el aprendizaje en torno a proyectos.

De acuerdo con las definiciones que se encuentran en los manuales de PBL para los profesores, los proyectos son tareas complejas, basados en preguntas o problemas desafiantes, que involucran a los estudiantes en el diseño, la resolución de problemas, toma de decisiones, o en actividades de investigación. Dan a los estudiantes la oportunidad de trabajar de forma relativamente autónoma durante largos períodos de tiempo; y culminan en productos realistas o presentaciones de resultados (Jones, Rasmussen, y Moffitt, (1997), Thomas, Mergendoller, y Michaelson (1999)).

El germen de este modelo de aprendizaje se halla en el constructivismo, que enfoca al aprendizaje como el resultado de construcciones mentales; esto es, que los seres humanos, aprenden construyendo nuevas ideas o conceptos, en base a conocimientos actuales y previos (Karlin y Vianni, (2001)).

Si bien el aprendizaje basado en proyectos es complicado y requiere gran dedicación y esfuerzo tanto por parte de los profeores como de los estudiantes, su proopuesta contribuye de manera primaria a (Galeano):

1. Crear un concepto integrador de las diversas áreas del conocimiento.

2. Promover una conciencia de respeto de otras culturas, lenguas y personas.

3. Desarrollar empatía por personas.

4. Desarrollar relaciones de trabajo con personas de diversa índole. 
5. Promover el trabajo disciplinar.

6. Promover la capacidad de investigación.

7. Proveer de una herramienta y una metodología para aprender cosas nuevas de manera eficaz.

Por lo que su aplicación deviene completamente defendible, de hecho recientes publicaciones ponen de manifiesto el esfuerzo que se está realizando en el desarrollo e implementación de esta metodología (Guerrero, Calero, (2013), Márquez, Jiménez-Rodrigo, (2014), Moral, Ballesteros, Tijero, Torrecilla, (2015), Izquierdo, Benítez, Berenguer, Lago-Alonso, (2016)).

\section{Objectivos}

Los principales objetivos de nuestro método alternativo de enseñanza de álgebra lineal son los siguientes:

a) Animar a los estudiantes a participar y formar parte del proceso de aprendizaje a través de enseñanzas interactivas y de debates que conducirán a los estudiantes a descubrir los conceptos principales por ellos mismos.

b) Fomentar el aprendizaje cooperativo. Los proyectos de equipo y los informes son excelentes vehículos para el aprendizaje cooperativo. Los estudiantes trabajan en conjunto para solucionar o resolver problemas que son importantes para ellos.

c) Introducir a los estudiantes en el arte de desarrollar y escribir expresiones matemáticas correctamente.

d) Ayudar a los estudiantes a pensar de una manera precisa y verbalizar sus pensamientos con claridad. Ayudar a la incorporación de software matemático para el cálculo de matrices, herramienta básica para el tratamiento del álgebra lineal.

e) Aprender a utilizar las herramientas de las TIC no sólo como un apoyo en el aprendizaje de álgebra lineal, sino también como una forma de transmitir los conocimientos adquiridos durante el curso.

El aprendizaje basado en proyectos ayuda a los estudiantes a lograr un aprendizaje significativo y provechoso, debido a que los conecta con su mundo fuera del aula, y les genera confianza en la realización de sus potenciales acciones 


\section{Metodología}

Es importante notar en contra de la opinión bastante generalizada, que la enseñanza en grupos grandes no implica que tenga que ser impartida de forma tradicional como una clase magistral. Por lo tanto, bajo esta convicción, hemos introducido alternativas de enseñanza en una asignatura de primer curso de estudios de grado de ingeniería. Entre estas alternativas hemos introducido la enseñanza basada en proyetos (PBL), el e-portafolio y la herramienta matemática Matlab.

Los estudiantes deben diseñar, planificar y llevar a cabo un amplio proyecto, cuyo producto debe ser mostrado públicamente en sus portafolios electrónicos que deben ser visibles (por lo menos) por los profesores y compañeros de clase.

En el marco que nos ocupa un e-portafolio es un método de enseñanza, aprendizaje y evaluación que consiste en la aportación de producciones de diferente tipo por parte del estudiante a través de las cuales se pueden juzgar sus capacidades con respecto una disciplina o materia de estudio.

El e-portafolio permite a los estudiantes crear y gestionar un espacio virtual con las actividades académicas, incorporando a su vez una valoración y justificación de la importancia que dichas actividades tienen.

Para poder implementar el e-portafolio es importante seleccionar el software a utilizar para la realización del e-portafolio, ya que no todas las plataformas permiten ser gestionadas con agilidad y sin demasiadas complicaciones técnicas, teniendo más o menos accesibilidad y mayor o menor visibilidad. Para que los estudiantes utilicen el portafolio de forma activa es necesario por parte del profesorado preparar pautas y directrices coherentes y consistentes, así como facilitar una plantilla de referencia que les pueda seguir de guía.

Para esta experiencia la UPC ha facilitado direcciones URL para todos los estudiantes dentro de su servidor y se ha utilizado la plataforma Wordpress 4.5.2 para diseñar los e-portafolios.

\subsection{Experimento}

Durante el año académico 2014-15, se inició una prueba piloto de aprendizaje colaborativo basado en proyectos por medio de e-portafolios en una asignatura de matemáticas básica concretamente de Álgebra lineal. Los participantes fueron los estudiantes de primer año de grado de la "Escuela Técnica Superior de Ingenieros Industriales de Barcelona, (ETSEIB)" en la "Universitat Politècnica de Catalunya, (UPC) ".

Referente al curso impartido durante el primer cuatrimestre había un total de 651 alumnos, distribuidos en 10 grupos denominados $G 1, \ldots, G 10$, no todos del mismo tamaño oscilando entre 50-85 alumnos por grupo (los de menor tamaño correspondían a grupos de tarde). Todos ellos trabajaron individualmente en sus e-portafolios, a la vez que realizaban la tarea en grupos de 4 estudiantes y excepcionalmente algunos 
de 5 en caso de que el número de alumnos de su grupo $G i$ no fuese múltiplo de 4, denominados Gij1 $\leq i \leq 10,1 \leq j \leq 5$. Estos subgrupos se han mantenido a lo largo de todo el curso.

Con respecto al curso impartido durante el segundo cuatrimestre el total de alumnos era de 122 repartidos en 3 grupos de entre 25-50 alumnos que al igual que el primer cuatrimestre el de menos tamaño corresponde al grupo (en este caso sólo uno) de tarde, y también realizaron la tarea en subgrupos de 4 estudiantes (excepcionalmente alguno de 5)

El principal objetivo de este proyecto es analizar si el uso de PBL puede ser eficaz para el aprendizaje del Álgebra Lineal. El hecho de utilizar casos prácticos ayuda a los estudiantes a que creen la necesidad de aprender los temas de la asignatura y esto se ha considerado un punto clave a tener en cuenta en el momento de poner en práctica el proyecto.

\subsubsection{Planificación de la asignatura}

\begin{tabular}{|c|c|}
\hline 1a semana & $\begin{array}{c}\text { Presentación del curso, explicación del e-portafolio } \\
\text { Formación de subgrupos en el aula y en el foro (Moodle) } \\
\text { Material de apoyo } \\
\text { Inicio e-portafolio }\end{array}$ \\
\hline 2a semana & $\begin{array}{c}\text { Presentación del proyecto } \\
\text { Explicación del enunciado } \\
\text { Descripción de las tareas individuales y grupales }\end{array}$ \\
\hline $3 a-5 a$ semana & $\begin{array}{c}\text { Proyecto: primera parte } \\
\text { Tutorización del trabajo } \\
\text { Seguimiento a través de Moodle } \\
\text { Rúbrica para autoevaluación }\end{array}$ \\
\hline 6a semana & $\begin{array}{l}\text { Entrega primera parte del proyecto } \\
\text { Evaluación del profesor mediante rúbrica }\end{array}$ \\
\hline 7a semana & $\begin{array}{c}\text { Proyecto segunda parte } \\
\text { Descripción de las tareas }\end{array}$ \\
\hline 7a-14a semana & $\begin{array}{c}\text { Tutorización del trabajo } \\
\text { Seguimiento a través de Moodle } \\
\text { Rúbrica para autoevaluación }\end{array}$ \\
\hline 15 a semana & $\begin{array}{l}\text { Entrega final proyecto } \\
\text { Evaluación del profesor mediante rúbrica } \\
\text { Rúbrica para evaluación por pares }\end{array}$ \\
\hline
\end{tabular}

El tiempo total dedicado al proyecto es de 150 horas de las cuales el $40 \%$ corresponden al trabajo realizado en el aula y el $60 \%$ restante al trabajo realizado fuera del aula. Las clases teóricas ocupan un $25 \%$ del tiempo dedicado a las tareas del aula, completando el tiempo con tutorías y resolución de las dudas que les han surgido a los estudiantes y a trabajar colaborativamente en el proyecto planteado en relación al contenido teórico de la asignatura. 


\subsubsection{Proyecto: Estudio de los procesos de servicios hospitalarios para a la toma de} decisiones en la administración de la salud

A continuación, detallamos la tarea a realizar por los estudiantes durante el segundo cuatrimestre.

Teniendo en cuenta la obligatoriedad de satisfacer las necesidades de los pacientes cuando se hallan recibiendo un servicio dentro de un centro hospitalario, se trata de determinar el tiempo medio de estancia de los pacientes en una sala determinada de dicho hospital, con un determinado nivel de probabilidad en estos estados transitorios.

Esta tarea consiste en buscar una herramienta para la toma de decisiones dentro del campo de la administración de la salud con el fin de optimizar los recursos disponibles.

El objetivo final de este proyecto es el estudio de los modelos de Markov útiles para el análisis tendencial o prospectivo del desarrollo tecnológico, como instrumento de cálculo de que un hecho suceda. Para esto se utilizan dos factores probabilísticos: la secuencia de los hechos y el tiempo transcurrido entre eventos sucesivos, esto es, la transición de estados y el tiempo de permanencia en el estado.

- Paso 1: Modelar la evolución de un determinado servicio de un centro hospitalario. Los pacientes una vez intervenidos quirúrgicamente pasan a la sala de recuperación (Estado $S_{1}$ ), donde se sabe que hay una probabilidad $\alpha_{11}$ de permanecer en ella, la de ir a casa (Estado $C$ ) es de un $\alpha_{1 c} \%$ y se conoce por estudios anteriores que hay un porcentaje $\alpha_{12}$ de pacientes que pueden pasar a la sala de terapia intermedia (Estado $S_{2}$ ) por complicaciones o por la propia patología del paciente y a la vez que el resto de pacientes pasan a terapia intensiva (Estado S3) por los mismos problemas. Los pacientes que están en la sala intermedia o permanecen en ella o tienen una probabilidad de un $\alpha_{21}$ de pasar a la sala de recuperación y un $\alpha_{23} \%$ de pasar a la sala de terapia intensiva. Finalmente, los pacientes de la sala terapia intensiva $S_{3}$ tienen una probabilidad $\alpha_{33}$ de permanecer en ella, un $\alpha_{32} \%$ de pasar a la sala de terapia intermedia y el resto de pacientes mueren (Estado $M$ ).

Los estudiantes deben modelar el problema en la forma

$$
S(k+1)=A S(k)
$$

más concretamente

$$
\left(\begin{array}{c}
S_{1}(k+1) \\
S_{2}(k+1) \\
S_{3}(k+1) \\
C(k+1) \\
M(k+1)
\end{array}\right)=\left(\begin{array}{ccccc}
\tilde{\alpha}_{11} & \tilde{\alpha}_{21} & 0 & 0 & 0 \\
\tilde{\alpha}_{12} & \tilde{\alpha}_{22} & \tilde{\alpha}_{32} & 0 & 0 \\
\tilde{\alpha}_{13} & \tilde{\alpha}_{23} & \tilde{\alpha}_{33} & 0 & 0 \\
\tilde{\alpha}_{1 c} & 0 & 0 & 1 & 0 \\
0 & 0 & \tilde{\alpha}_{34} & 0 & 1
\end{array}\right)\left(\begin{array}{c}
S_{1}(k) \\
S_{2}(k) \\
S_{3}(k) \\
C(k) \\
M(k)
\end{array}\right)
$$




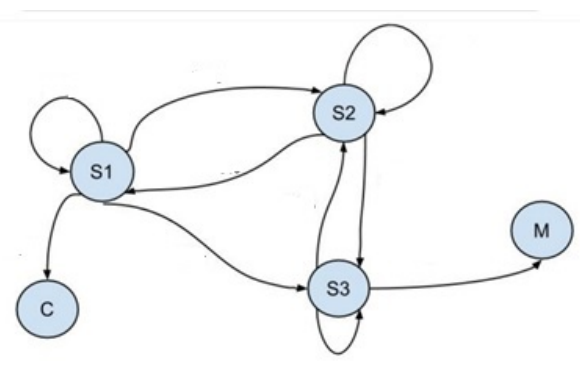

Fig. 1: Grafo

con $\tilde{\alpha}_{i j}=\alpha_{i j} / 100$ y $\tilde{\alpha}_{13}=1-\left(\tilde{\alpha}_{11}+\tilde{\alpha}_{12}+\tilde{\alpha}_{1 c}\right), \tilde{\alpha}_{22}=1-\left(\tilde{\alpha}_{21}+\tilde{\alpha}_{23}\right)$, $\tilde{\alpha}_{34}=1-\left(\tilde{\alpha}_{32}+\tilde{\alpha}_{33}\right)$.

Para la obtención del modelo se pueden ayudar construyendo un grafo como el que se muestra en la figura 1.

En este momento los estudiantes deben preguntarse acerca de la solución del sistema de ecuaciones dinámico y para ello, deben encontrar inductivamente la siguiente ecuación.

$$
S(k)=A^{k} S(0)
$$

Y ahora, los estudiantes necesitan aprender a manejar las operaciones con matrices y, especialmente, necesitan aprender cómo calcular las potencias de la matriz. Cuando los estudiantes llegan a este punto, en las siguientes clases, el profesor explica la teoría de matrices, introduce los espacios vectoriales y las aplicaciones lineales. Además, el profesor invita a los estudiantes a empezar a utilizar MATLAB con la guía claro está, del profesor.

- Paso 2: Explicar con todo detalle la información requerida de Álgebra Lineal para resolver la ecuación, como por ejemplo (ver Garcia y Dominguez (2014) para más detalles):

a) Analizar el tipo de matriz que describe el sistema.

Los estudiantes observan que la matriz es estocástica y además es triangular por bloques con un bloque de la diagonal igual a la identidad.

b) Cómo calcular potencias de una matriz, en vistas a analizar si hay una distribución sostenible de la utilización de las salas del hospital.

Los estudiantes observan que si la matriz es diagonal es muy fácil obtener las potencias de dicha matriz, y los profesores esperan que los estudiantes relacionen lo que se ha estado explicando en clase, (en este caso, las explicaciones sobre la semejanza de matrices $\left.\left(A=S D S^{-1}\right)\right)$, con su problema de la matriz estocástica. 
En esta altura de curso, el profesor explica diagonalización de endomorfismos y la forma reducida de Jordan.

También comprueban que para el caso particular que les ocupa de la matriz triangular pueden reducir los cálculos a matrices de orden menor pues

$$
A^{n}=\left(\begin{array}{ll}
T & 0 \\
Q & I
\end{array}\right)^{k}=\left(\begin{array}{cc}
T^{k} & 0 \\
Q\left(\sum_{i=0}^{k-1} T^{i}\right) & I
\end{array}\right)
$$

A partir de este momento se dan valores concretos a los parámetros $\alpha_{i j}$.

- Paso 3: Se trata ahora de averiguar la probabilidad de que un paciente que se encuentra en la sala de terapia intensiva, pueda estar en su casa al cabo de cuatro días

- Paso 4: Obtención de los valores propios y análisis de la estabilidad.

Los estudiantes calculan los valores propios y observan que en módulo son todos menores o iguales que 1.

Después de recoger toda la información obtenida hasta el momento, se supone que los estudiantes observan que

$$
\sum_{i=0}^{\infty} T^{i}=\lim \sum_{i=0}^{k-1} T^{i}=(I-T)^{-1}
$$

y que llamando $u(k)=\left(S_{1}(k), S_{2}(k), S_{3}(k)\right)^{t}, v(k)=(C(k), M(k))^{t}$ se tiene $\lim u(k)=0, \lim v(k)=v(0)+Q(I-T)^{-1} u(0)$.

En este momento, los estudiantes usan MATLAB para el cálculo matricial a realizar, cuyo manejo (nivel principiantes) se les ha enseñado durante el curso teórico.

Es importante poner todas las referencias utilizadas (libros, páginas web y comentarios con otros grupos ...) en todas las etapas del proyecto. La última entrega se realiza durante la última semana del curso. En esta fase final, los estudiantes también entregan el e-portafolio que debe contener su currículo y su propia visión general del tema. Obviamente, cada parte de trabajo que se ha ido actualizando en el e-portafolio ha sido revisado y se le ha dado la retroacción por parte del profesor e incluso por sus compañeros. 


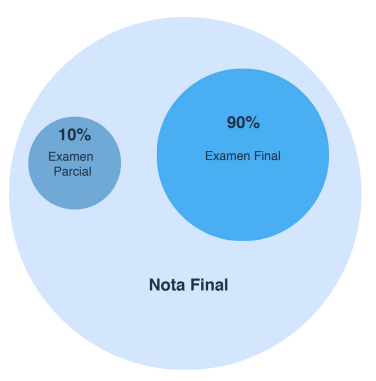

Fig. 2: Evaluación curso 2013-14,

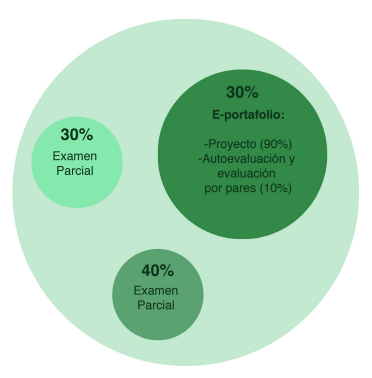

Evaluación curso 2014-15

\subsection{Evaluación}

La evaluación del proyecto tal y como se ha indicado en la planificación, se realiza mediante rúbricas. La nota obtenida tiene un peso del $30 \%$ de la nota final de la asignatura. El resto de nota se obtiene mediante exámenes que obliga el centro a realizar. La normativa obliga a la realización de un examen parcial a mediados del cuatrimestre y otro a final de curso, representando el $30 \%$ y $40 \%$ respectivamente, de la nota final.

En los cursos anteriores a la implementación del PBL, la evaluación (ver figura 2) se efectuaba mediante dos exámenes uno a mediados del cuatrimeste con un peso del $10 \%$ de la nota final y un examen final con un valor del $90 \%$, dando toda la carga de la nota de la asignatura al examen final.

\section{Resultados}

Hemos comparado las diferentes calificaciones de los estudiantes durante los períodos que corresponden a los cursos 2013-14 y 2014-15, este último es el período en el que se ha implementado el PBL e introducido el e-portafolio como parte del plan de estudios, (ver Figuras 3, 4, 5, 6).

Se ha reducido el número de estudiantes que no supera la asignatura en un 16,68\% en el primer semestre del 2014-15, con respecto al mismo semestre en el curso 2013-14. Más significativos son los datos aún si los comparamos con el segundo semestre de los respectivos cursos, en este semestre se matriculan solamente los estudiantes que no superaron la asignatura en el primer semestre, se produce una reducción del 37,91\%. En global en el curso 2014-15 sólo un 2,33\% de los alumnos que se matricularon en el mes de setiembre no consiguieron superar la asignatura.

Se observa claramente, la mejora de las calificaciones y la reducción del fracaso. 


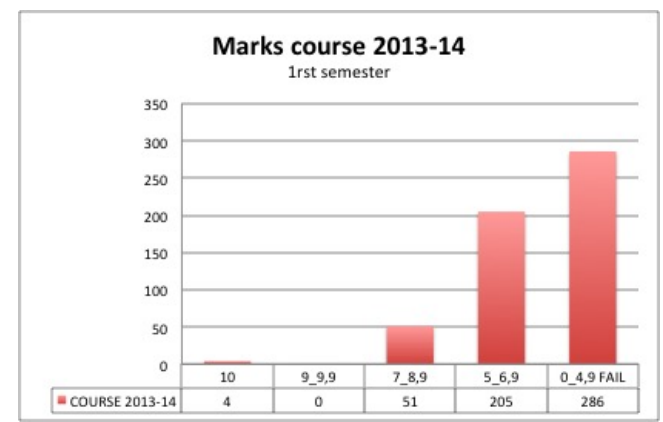

Fig. 3: Calificaciones curso 2013-14 primer semestre

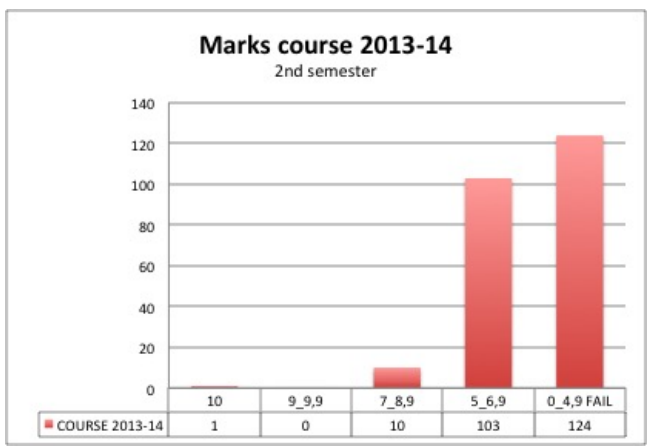

Fig. 4: Calificaciones curso 2013-14 segundo semestre

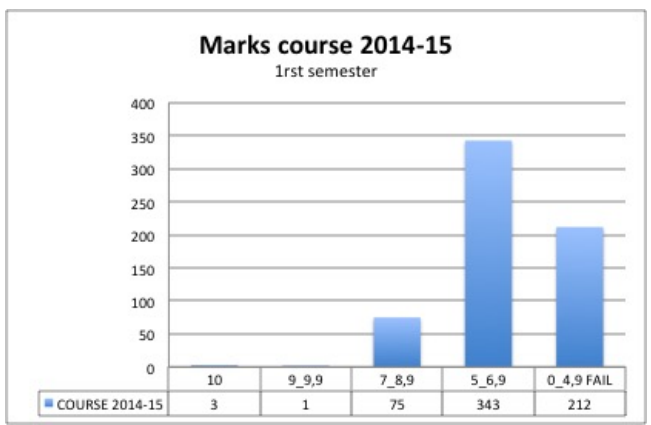

Fig. 5: Calificaciones curso 2014-15 primer semestre 


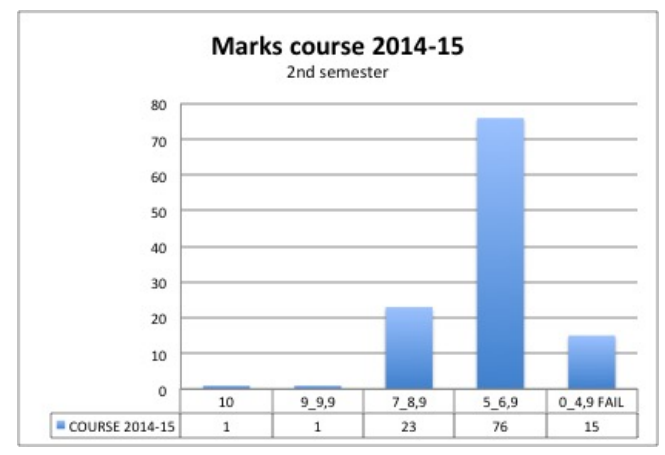

Fig. 6: Calificaciones curso 2014-15 segundo semestre

\section{Conclusiones}

Con la implementación del aprendizaje basado en proyectos y el uso del e-portafolio se ha puesto de manifiesto un claro aumento de la motivación y de la mejora de la autonomía de los estudiantes. Ellos han estado trabajando de forma individual y en grupos, por lo que también han sido capaces de cooperar y trabajar en euipo para resolver las diferentes tareas relacionadas con el proyecto.

Con esta experiencia también se ha conseguido mejorar los resultados académicos de los esudiantes y con el papel más activo que han tenido han podido alcanzar las competencias tanto específicas como genéricas del álgebra lineal con un cierto nivel de exigencia. La resolución de casos prácticos ha servido para mejorar la comprensión de los contenidos de la materia y han utilizado el e-portafolio como una herramienta para optimizar dicha comprensión ya que han debido aprender a estructurar, organizar, comunicar y presentar los trabajos realizados.

La información sobre las fortalezas y debilidades sobre la asignatura que los alumnos reciben a través de la rúbrica de autevaluación ha infuido muy positivamente en ellos, haciendo que desarrollen capacidades que con otro tipo de evaluciones no se habían conseguido.

Después de los resultados obtenidos se puede concluir que los objetivos de mejora de la comprensión de la asignatura, con la introducción del PBL han sido alcanzados. Aunque haya supuesto un duro trabajo de preparación, la experiencia ha sido muy positiva y merece la pena seguir trabajando en ella. 


\section{Referencias bibliográficas}

1. Aydin, A. The factors effecting teaching linear algebra. World Conference on Educational Sciences. Procedia Social and Behavioral Sciences, 1, 2009. p. $1549-1553$.

2. Bri, D., García, M., Coll, H. LLoret, J. A Study of Virtual Learning Environments. Wseas Transactions on Advances in Engineering Education, 6(1), 2009. p. $33-43$.

3. Brookhart, S.M. The Art and Science of Classroom Assessment: The Missing Part of Pedagogy. ASHE-ERIC Higher Education Report Washington, DC: The George Washington University, Graduate School of Education and Human Development, 27(1). 1999.

4. Carlson, D. C.R. Johnson, D.C. Lay, A.D. Porter. The linear algebra curriculum Study group recommendations for the first course in linear algebra. The College Mathematics Journal, 24(1), 1993. p. 41-46.

5. Domínguez-García, S. Creació d'una rúbrica per avaluar la competència docent en l'ús de la Pissarra Digital Interactiva, TFG. Universitat Rovira i Virgili. 2014.

6. Domínguez-García, S., García-Planas, M.I., Palau, R., Taberna, J. Using the e-portfolio for large groups of students Proceedings of INTED2015 Conference 2015, p. 1352-1360.

7. Galeano de la O., L. Aprendizaje basado en proyectos http://ceupromed.ucol.mx/ revista/PdfArt/1/27.pdf,

8. García-Planas, M.I., Domínguez, J.L. Introducción a la teoría de matrices positivas. Aplicaciones. Ed. Iniciativa Digital Politècnica, Barcelona, 2013.

9. Guerrero, E., Calero, J. El aprendizaje basado en proyectos como base metodológica en el grado de Educación Social Educación social. Revista de Intervención Socioeducativa 53, 2013. p. 73-91

10. Harel, G. Principles of Learning and Teaching Mathematics, With Particular Reference to the Learning and Teaching of Linear Algebra: Old and New Observations. In J-L. Dorier Ed. On the Teaching of Linear Algebra, Kluwer Academic Publishers, Dordrecht. 2000. p. 177-189,

11. Izquierdo. J, Benítez, J. Berenguer, A , Lago-Alonso, C. I decide, therefore I am (relevant!): A project-based learning experience in linear algebra. Computer Applications in Engineering Education. 24, (3), p. 481-492, 2016

12. Jones, B.F., Rasmussen, C. M., \& Moffitt, M. C. (1997). Real-life problem solving.: A collaborative approach to interdisciplinary learning. Washington, DC: American Psychological Association. 
13. Karlin, M., Viani, N. (2001). Project-based learning. Medford, OR: Jackson Education Service District.

14. Koh, J.H.L. A rubric for assessing teachers' lesson activities with respect to TPACK for meaningful learning with ICT. Australasian Journal of Educational Technology, 29(6), 2013. p. 887-900.

15. Márquez, E. y Jiménez-Rodrigo, M.L. El aprendizaje por proyectos en espacios virtuales: estudio de caso de una experiencia docente universitaria. Revista de Universidad y Sociedad del Conocimiento (RUSC). 11(1). (2014). p. 76-90. Doi http://dx.doi.org/10.7238/rusc.v11i1.1762

16. Moral, A., Ballesteros, M., Tijero, A., Torrecilla, J.S. Estrategias metodológicas para el aprendizaje basado en proyectos de investigación en Ingeniería de Bioprocesos. International Journal of Educational Research and Innovation. 4, (2015). p. 91-101.

17. Thomas, J. W., Mergendoller, J. R., and Michaelson, A. (1999). Project-based learning: A handbook for middle and high school teachers. Novato, CA: The Buck Institute for Education.

18. Yildiz, A.U. Teaching the diagonalization concept in linear algebra with technology: A case study at Galatasaray University. The Turkish Online Journal of Educational Technology, 12(1), 2013. p. 119-130, 